\title{
Serum Surfactant Protein-D (SP-D) Level: Does it Correlates with Pulmonary Compliance and ARDS in Severe Cases of COVID-19?
}

\author{
Jayadi $^{*}$, Prananda Surya Airlangga ${ }^{1}$, Edward Kusuma ${ }^{1}$, Christrijogo Soemartono \\ Waloejo $^{1}$, Agustina Salinding ${ }^{1}$, Pudji Lestari ${ }^{2}$ \\ 1Departement of Anesthesiology and Reanimation, Medical Faculty of Airlangga University, Dr. Soetomo General Hospital, Surabaya, Indonesia \\ 2Departement of Public Health and Preventive Medicine, Medical Faculty of Airlangga University, Surabaya, Indonesia \\ *Email: jayadiiipang@gmail.com
}

\begin{abstract}
Acute Respiratory Distress Syndrome (ARDS) happens to 67\% of all severe COVID-19 cases with mortality rate of 50-94\%. Main characteristics of ARDS are poor oxygenation and non-compliant lungs. The lungs are like inflatable balloons that are actively inflated by the positive pressure within and/or the negative pressure created in the pleural cavity. Compliance is the ability of the lungs to distend at a certain level of transpulmonary pressure.

Surfactant lowers the surface tension of the alveoli so that end-expiratory pulmonary collapse could be prevented and pulmonary compliance could be maintained. Surfactants also play an important role in the host's defense against infection.

Surfactants comprise of phospholipids, cholesterol and surfactant proteins (SP) SP-A, -B, -C and -D. Previous study showed that concentration of SP-D was significantly higher in ARDS patients. Higher serum SPD concentrations were associated with a greater risk of death, fewer ventilator-free days, and fewer organ failure-free days. This review highlights the role of surfactant especially SP-D in pulmonary compliance and ARDS in COVID-19.
\end{abstract}

Keywords: surfactant; pulmonary compliance; ARDS; severe COVID-19

\section{Introduction}

COVID-19 could manifest as a severe case. Acute Respiratory Distress Syndrome (ARDS) takes up to $67 \%$ of all severe COVID-19 cases with mortality rate of 50-94\%. Berlin's criteria for ARDS includes (1) acute hypoxemic respiratory failure due to certain cause, (2) appearance of bilateral infiltration in thorax computed tomography scan (CT scan) or thorax plain radiograph, (3) not fully attributed by cardiogenic failure and/or volume overload [1].

ARDS is a syndrome of a pathologic condition with main characteristics of poor oxygenation and noncompliant lungs. ARDS is caused by acute and diffuse inflammation of the lungs that makes increase in pulmonary vascular permeability, pulmonary resistance, and loss of aerated lung tissues with hypoxemia and bilateral opacities in imaging [2]. 
The prominent clinical presentation of ARDS is decreased pulmonary compliance. Definition of pulmonary compliance is a change of volume that occurs for each unit of pressure change in the respiratory system [3]. One of the factors that play a role in pulmonary compliance is elastin and collagen fibers, because of which lungs tissues have elastic properties and tend to be deflated and stiffened. The decrease in pulmonary compliance in ARDS is not caused by stiffened tissues, but by the hydrostatic pressure of interstitial edema which compresses and collapses the alveoli. In lungs with ARDS, the alveoli tend to collapse because of the accumulation of inflammatory cells and fluid in the interstitial spaces of the alveoli, as well as reduced function of surfactant [4].

Surfactant plays a role in maintaining pulmonary compliance by decreasing the surface tension of the alveoli so that end-expiratory pulmonary collapse could be prevented. Surfactant also has a role in eradicating or preventing spread of pathogens and modulating immune responses. Surfactant is consisted of phospholipids and surfactant proteins (SP) SP-A, -B, -C and -D [5, 6].

Surfactant protein D (SP-D) is a member of the family of collectins. It is involved in innate immunity of the host and modulate the adaptive immune system. SP-D is mainly produced by type II alveolar cells. Normally, Pulmonary collectins are expressed exclusively in the lungs. Any leaks to the circulation is a response to increased pulmonary vascular permeability due to damage of structural and functional integrity [7]. This condition is accurately reflected in COVID-19 pneumonia. Higher serum SP-D levels were found in severe cases of COVID-19, especially in the acute phase [8, 9]. Serum SP-D levels were positively related to C-Reactive Protein (CRP), duration of the oropharyngeal swab from positive to negative, initial thorax CT score at hospital admission, and hospital length of stay (LoS) [9].

\section{Pulmonary Compliance}

The lungs are like inflatable balloons that are actively inflated by the positive pressure within and/or the negative pressure created in the pleural cavity. The term respiratory mechanics describes the mechanical properties of the respiratory system that increase during mechanical ventilation. Monitoring of respiratory mechanics is useful for diagnosing and evaluating lung conditions, severity of lung disorders, and adjusting the ventilator settings. One parameter that can be seen is pulmonary compliance.

Compliance is defined as the ability of the lungs to distend at a certain level of transpulmonary pressure (Ptp). In normal breathing, negative pleural pressure $(\mathrm{Ppl})$ is sufficient to stretch the lungs during the inspiration phase [10]. Distension pressure or transpulmonary pressure is calculated using the equation (1):

$$
\begin{array}{ll}
\text { Ptp = Palv }- \text { Ppl } \\
\text { Ptp } & =\text { transpulmonary pressure } \\
\text { Palv } & =\text { alveolar pressure } \\
\text { Ppl } & =\text { pleural pressure }
\end{array}
$$

Pulmonary compliance (C) is usually used to measure change in lung volume which is the extent of the lungs expansion for each unit increase in transpulmonary pressure (when sufficient time is available to reach equilibrium) [3]. Pulmonary compliance can be calculated using the formula (2): 
$\mathrm{C}=\mathrm{V} /(\mathrm{Pal}-\mathrm{Ppl})$

$\mathrm{C} \quad=$ pulmonary compliance

$\mathrm{V}=$ change in lung volume

Palv = alveolar pressure

Ppl = pleural pressure

Compliance depends on lung tissue elasticity, surface tension of elastic forces, surfactant, lung volume, and age [10]:

\subsection{Lung tissue elasticity}

Lung elasticity comes from collagen and elastin fibers fused in the pulmonary parenchyma. When the lungs are outside the body's system and in a deflated state, these fibers contract completely due to elasticity. When the lungs expand, they lengthen and exert a more elastic force like a rubber band. The elasticity of these fibers determines lung compliance. They can be damaged or affected by certain lung pathologies [5].

\subsection{Surface tension of elastic forces}

The flexible nature of the lung is determined not only by the elastic strength of the tissue but also by the contribution of the surface tension that comes from the fluid lining the walls of the alveoli [11]. Water molecules have a strong attractive force for each other when they forms a surface with air, causing surface tension forces. The water that coats the inner surface of the alveolus will force air out of the alveolus and deflate it. This force is called the surface tension of elastic force. The minimum value is $35-41 \mathrm{dyne} / \mathrm{cm}$. Thus, surface tension elastic forces are antagonistic to lung compliance [12].

\subsection{Surfactant}

The Laplace's law can be used to measure alveolar pressure by using the surface tension exerted by the fluid layer according to the formula: Pressure $=2 \times \mathrm{T}$ (Surface Tension) / $\mathrm{R}$ (Radius). The pressure in the smaller alveolus is higher than that of the large alveolus. However, in the general scenario, this is not the case because of the surfactant. Surfactant is an active substance in fluid, which is secreted by type II alveolar epithelial cells. Surfactant contains dipalmitoylphosphatidylcholine phospholipid, surfactant apoprotein, and calcium ions. Surface tension is reduced because of the surfactants by partial decomposition. Smaller alveoli have a small surface area so that the surfactant concentration is higher. The surface tension remains the same even though the pressure in the alveolus is higher. Because of its role in modification of surface tension, it indirectly affects pulmonary compliance [5].

Type I alveolar cells or squamous cells lining the barrier between the air space and the septal wall, together with a thin layer of surfactant and capillary endothelial cells, form the alveolar septum which acts as a barrier between air and blood [13].

\subsection{Lung volume}

Compliance correlates to lung volume, this is indicated by the lowest compliance at extreme functional residual capacity (FRC) [14]. This implies that the expanded lung and the contracted lung have a lower capacity to distend at a given pressure. 


\subsection{Age}

The age factor has the least effect on pulmonary compliance. Compliance increases with age as structure of lung elastic fibers changes [15].

Based on the measurement method, pulmonary compliance can be categorized as static or dynamic [16]. Dynamic compliance (Cdyn) is a continuous unit of lung compliance which is calculated at each point representing a schematic change during rhythmic breathing. Cdyn monitors elastic resistance and airway resistance. Air density, density, length, and radius of the airway determine airway resistance. All of those variables are relatively constant except the airway radius. Thus, changes in the radius of the bronchial airways have a role in physiological changes of airway resistance [3].

Static compliance or usually abbreviated as Cstat, represents compliance of the lungs at a certain fixed volume in certain conditions. Those conditions include no airflow and relaxed muscles. This situation occurs when the transpulmonary pressure and elastic recoil pressure of the lungs are equal. Cstat only measures elastic resistance. Measurement is done using a manometer, however electrical transducers are now a more common choice. In conscious patients, complete relaxation of the respiratory muscles is difficult to be achieved. However the measurement can be considered valid because the difference in static pressure is not affected by muscle activity. In paralyzed individuals (e.g. in the operating theatres), static compliance is easy to be measured using recordings captured via electrical transducers. Therapeutically, it is used to select the appropriate level of positive end-expiratory pressure (PEEP) using the following formula (3) [3]:

$$
\text { Cstat }=\mathrm{V} /(\text { Pplat }- \text { PEEP })
$$

$$
\begin{aligned}
& \text { Cstat }=\text { static compliance } \\
& \mathrm{V}=\text { change in lung volume } \\
& \text { Pplat }=\text { plateau pressure } \\
& \text { PEEP }=\text { Positive End Expiratory Pressure }
\end{aligned}
$$

The study conducted by Arnal, et al. involving ARDS subjects with decreased pulmonary compliance, normal subjects, and subjects with COPD with increased pulmonary compliance. Result was pulmonary compliance could be categorized based on its Cstat level into low compliance (Cstat $<50 \mathrm{ml} / \mathrm{cmH} 2 \mathrm{O}$ ), normal compliance (Cstat 50-60 ml/cmH2O) and high compliance (>60 ml/cmH2O) [17].

\section{Acute Respiratory Distress Syndrome (ARDS)}

Acute respiratory distress syndrome (ARDS) is a collection of clinical and physiological manifestations that describe a pathological condition. ARDS happens when there is an injury to the lung due to an acute and diffuse inflammatory process, which results in increased pulmonary vascular permeability, increased pulmonary resistance, and loss of aerated lung tissue, with hypoxemia and bilateral opacity on chest imaging. It is associated with increased shunting, increased physiologic dead space, and reduced pulmonary compliance. The damage occurs due to local production of proinflammatory mediators or those distributed through the pulmonary arteries. This causes loss of alveolar-capillary barrier integrity resulting in transudation of protein-rich edema fluid [2].

There are two types of alveolar cells, namely type I and type II cells. Type I cells are easier to be damaged, leading to the entry of fluid into the alveoli and decreased clearance of fluid from the alveolar cavities. On the other hand type II cells have multiple roles such as surfactant production, ion transport, proliferation and 
differentiation into type I cells after trauma. Damage to these two cells leads to decreased surfactant production and decreased compliance [2].

ARDS manifestations vary depending on comorbidities, the degree of lung injury, and the presence or absence of other organ dysfunction. Symptoms include shortness of breath, requiring more effort to inhale, and hypoxaemia. Bilateral infiltrates on chest X-ray represent pulmonary edema. Multiple organ dysfunction syndrome (MODS) can occur due to systemic biochemical abnormalities. The time limit for ARDS is one week from the appearance of a new onset or from worsening of a respiratory symptom [2].

Berlin's definition for ARDS includes (1) acute onset that is within one week of known clinical insult on new or worsening respiratory symptoms, (2) bilateral opacities, not fully explained by effusions, lobar/lung collapse, or nodules, (3) respiratory failure, not fully explained by cardiac failure or fluid overload, (4) $\mathrm{PaO} 2 / \mathrm{FiO} 2 \leqslant 300 \mathrm{mmHg}$ with PEEP or CPAP $\geqslant 5 \mathrm{cmH} 2 \mathrm{O}$. Factors that affect mortality in ARDS include age greater than 65 years, chronic liver disease, and multiple organ dysfunction [18].

\section{ARDS in COVID-19}

Acute lung injury (ALI) that progresses rapidly to ARDS is the leading cause of high mortality in pneumonia, including SARS-CoV-2-associated pneumonia. The transition of ALI to ARDS happens due to diffuse alveolar damage, usually as a consequence of an exaggerated and uncontrolled systemic inflammatory reaction [19].

In the early stages of ALI, activation of antigen presenting cells (APCs) like macrophages and dendritic cells triggers immune response and facilitates the production of pro-inflammatory cytokines (mainly TNF $\alpha$, IL-1, and IL-6), as well as prostaglandins and histamine. These substances increase permeability of endothelium and facilitate migration of neutrophils, macrophages, and lymphocytes to location of inflammation, which exacerbates injury and triggers ARDS [19].

Edema caused by damage to type II pneumocytes has effects on production of surfactants and inflammatory mediators. Production of surfactant decreases, on the other hand production of inflammatory mediators (eg pro-inflammatory cytokines IL-1 $\beta$, IL-6, and IL-18) increases. These mediators stimulate homing of macrophages and neutrophils to the lung by chemotaxis. The formation of hyaline membranes in the alveoli takes place during this phase. The presence of multinucleated giant cells that are macrophages and pneumocytes indicates the involvement of macrophages in pathogen phagocytosis. The exudative phase of ARDS ends with a transition to a proliferative (rehabilitation) phase characterized by regeneration of pneumocytes and endothelium under conditions of suppressed proinflammatory cytokine production. When neutrophils are massively killed, macrophages complete debridement sites by phagocytosis and their capacity for production of anti-inflammatory factors (e.g. IL-10 and TGF- $\beta$ ) is increased [19].

The over-inflammatory state is determined primarily by pro-inflammatory cytokines. Three of the most important pro-inflammatory cytokines of the innate immune response are IL-1, TNF- $\alpha$, and IL-6 [20]. Tissue macrophages, mast cells, endothelium, and epithelial cells are the main sources of these cytokines during the innate immune response. A 'cytokine storm' results from an acute increase in various pro-inflammatory cytokines including IL-6, IL-1, TNF $\alpha$-, and interferons in the circulation. This increase in cytokines results in the entry of various immune cells such as macrophages, neutrophils, and T cells from the circulation to the site of infection with destructive effects on human tissues due to destabilization of endothelial cell-to-cell interactions, damage to the blood vessel barrier, capillary damage, diffuse alveolar damage, multiorgan failure, and finally death [19]. 
ARDS caused by COVID-19 appears to be different in many ways from other types of ARDS. For example, many COVID-19 patients initially present with hypoxaemia with maintained pulmonary compliance and low elasticity, which is called type L presentation. Characteristics of L-type ARDS are (1) low elasticity with nearly normal compliance which means that the amount of gas in the lungs is close to normal, (2) low ventilation-to-perfusion (VA/Q) ratio, (3) low lung weight, (4) low recruiting power of the lungs. These Ltype patients can often be treated with oxygen supplementation and the prone position, or if there is severe hypoxia, intubation with mechanical ventilation at a low PEEP may be needed [19].

However, L-type patients often turn into H-type that exhibit high elasticity and low compliance. Characteristics of H-type ARDS are (1) igh elasticity: ecreased gas volume due to increased edema causes increased elasticity of the lungs, (2) high right-to-left shunt, (3) high lung weight, (4) high lung recruit ability, it is associated with an increase in the amount of non-aerated tissue, as in severe cases of ARDS, leading to increased recruiting power. H-type patients usually require mechanical ventilation at higher PEEP. Low pulmonary compliance indicates worsening disease with manifestations of dyspnea and more severe hypoxia [21].

\section{Surfactant}

Pulmonary surfactants are monomolecular films consisted of complex mixtures of lipids and proteins that coats the alveolar air-liquid interface. The main functions of surfactants are (1) reducing the surface tension at the air-liquid interface thus collapse of alveoli at the end of expiration could be prevented, (2) killing pathogens or preventing their spread, and (3) modulating immune responses [22].

The surfactants comprise of $80 \%$ phospholipids, $10 \%$ cholesterol and 2-5\% surfactant proteins (SP) SP-A, $-\mathrm{B},-\mathrm{C}$ and $-\mathrm{D}$. The complex phospholipid composition ensures function against dynamically changing airliquid surface area during respiration. As much as $80 \%$ of phospholipids are PC, and 10-15\% are anionic phospholipids such as phosphatidylglycerol [23].

The Young-Laplace equation shows that lower surface tension is important not only for the alveoli, but also for other low-diameter structures within the lung. The high surface tension in the alveoli makes surfactants spread from the loci of secretion in the alveoli to all surfaces of the lung thus stabilizing the small respiratory tract as well as the alveoli [24].

Surfactants also play an important role in the host's defense against infection. Collectins SP-A and SP-D increase the elimination of bacteria and viruses [22]. The C-terminal lectin domain of this protein binds to pathogenic oligosaccharides found in viruses and bacteria. Roles of collectins in are: (1) opsonization of pathogens, (2) phagocytosis facilitation of pathogens by innate immune cells (e.g. macrophages and monocytes), and (3) regulation of mediators production [25, 26]. Previous studies showed that SP-A-deficient mice exhibit impaired eradication of bacteria and viruses [27, 28, 29, 30]. Recent study showed that SP-A and SP-D have direct antibacterial activity and antifungal activity against certain bacteria and fungi, through increased microbial membrane permeability [31,32].

Apart from facilitation and activation of the immune system, pulmonary collectins also has immunomodulatory function. SP-A inhibits dendritic cell maturation and release of eosinophils from IL-8 [33, 34]. Both SP-A and SP-D are involved in the mechanism against pathogen and both are potential prognostic factors. However, the study by Eisner et al. showed that the SP-A level was not associated with the clinical outcome of ARDS patients. Whereas higher serum SP-D concentrations were associated with a greater risk of death, fewer ventilator-free days, and fewer organ failure-free days [35]. 
One of the main effects of SP-D is the aggregation and enhancement of microbial and host cell phagocytosis. Beside lung tissue, this protein is found on the external or luminal surfaces of the respiratory tract, digestive tract, glands, reproductive tract, urinary tract, vascular and glandular epithelium. This is consistent with the role of SP-D in pathogen recognition, as most of the sites where it is expressed are on surfaces exposed to the external environment or with plasma, urine, tears, cerebrospinal fluid, and amniotic fluid, where the maintenance of a sterile environment is important. One of the main effects of SP-D is eradication of bacteria, viruses, fungi, and parasitic worms by binding and opsonization that aids phagocyte recognition. SP-D also initiates phagocytosis by binding to various inhaled pathogens, saccharides, lipids, and nucleic acids with broad specificity [36].

\section{Correlation Between Surfactant Protein-D and Pulmonary Compliance}

Pulmonary surfactants reduce surface tension thus maintain patency of alveolar at the end of expiration. The surfactant layer is exposed to the higher oxygen pressure than any other mucosal surface in the body and exposed to a variety of oxidizing toxins like ozone and metals contained in the air we breathe every day. Surfactant oxidation in humans and experimental animals leads to alveolar collapse, decreased lung compliance, and impaired gas exchange. Phospholipids and macrophages are protected from oxidative damage by the hydrophilic SP-A and SP-D. They block thiobarbituric acid reactive substances and conjugated dienes accumulation during the copper-induced or low-density lipoprotein particle induced oxidation of surfactant lipids by mechanisms that do not involve metal chelation or oxidatively modified proteins. Oxidation of low-density lipoprotein oxidation is instantly neutralized by SP-A or SP-D, indicating direct inhibition of free radical formation or propagation [37].

SP-D deficient transgenic mice exhibit foamy macrophages that increase $\mathrm{H} 2 \mathrm{O} 2$ production and accumulation of matrix metalloproteinases (MMPs) in the alveoli. Alveolar dilatation was observed in SP-Ddeficient mice at 3 weeks postnatal, resulting in emphysematous lungs [38].

\section{Correlation Between Surfactant Protein-D and ARDS}

SP-D is normally secreted in various parts of the body, but ARDS patients have significantly higher concentrations $(p=0.001)$ [39]. SP-D had been used as a prognostic biomarker for interstitial lung disease (ILDs) and ARDS $[8,9]$.

Type II alveolar cells and Club cells express the ACE2 receptor [40], which is the target of SARS-CoV-2 to enter cells [41]. Type II alveolar cells and Club cells increase the release of SP-D which is an acute phase reactant in response to infectious agents to maintain airway immunity. In a hyper-inflammatory state in SARS-CoV2 patients, an increase in SP-D which is positively correlated with an increase in IL-6 indicates a synergistic effect in an effort to fight the pathogen [42].

SP-D promotes neutralization and elimination of inflammatory triggering factors (i.e. bacterial or viral pathogens) so that pro-inflammatory cytokines for example IL-6 will decrease [43]. The opposite occurs in ARDS patients, where the inflammatory process and pro-inflammatory cytokine release are continuously increased with the aim of clearing pathogens but causing lung injury. Thus, in SARS-CoV2 patients with ARDS, the concentrations of SP-D and IL-6 appear to be higher than in patients without ARDS and increase with disease progression [39]. 
Pulmonary collectins, including SP-D, leak into the circulation in response to increased pulmonary vascular permeability due to loss of structural and functional integrity [7]. This condition is similar to the pathophysiology of COVID-19 pneumonia. Furthermore, structural integrity of the alveolo-capillary membrane breakdown leads to increase serum concentration of SP-D because of protein translocation. An increase in the serum SP-D level indicates lung injury. The cumulative effect of increased SP-D production and vascular leakage results in increased serum SP-D concentrations in ARDS patients [44].

\section{Conclusion}

SP-D protects phospholipids and macrophages from oxidative damage. Consequences of surfactant oxidation are alveolar collapse, decreased lung compliance, and impaired gas exchange. Concentrations of SP-D appears to be higher in COVID-19 patients with ARDS and increases with disease progression.

\section{Acknowledgements}

None.

\section{References}

1. Jafari, D., Gandomi, A., Makhnevich, A., Qiu, M., Rolston, D. M., Gottesman, E. P., Tsegaye, A., Mayo, P. H., Stewart, M. E., Zhang, M. and Hajizadeh, N. Trajectories of Hypoxemia \& Respiratory System Mechanics of COVID-19 ARDS in the NorthCARDS dataset. medRxiv, 2021. doi: 10.1101/2021.01.26.21250492.

2. Diamond, M., Peniston, H. L., Sanghavi, D. and Mahapatra, S. Acute Respiratory Distress Syndrome. StatPearls, 2021. Available at: https://www.ncbi.nlm.nih.gov/books/NBK436002/.

3. Desai, J. and Moustarah, F. Pulmonary Compliance. StatPearls, 2021. Available at: https://www.ncbi.nlm.nih.gov/books/NBK538324/.

4. Russotto, V., Bellani, G. and Foti, G. Respiratory mechanics in patients with acute respiratory distress syndrome. Annals of translational medicine, 2018;6(19), p. 382. doi: 10.21037/atm.2018.08.32.

5. Morgan, T. E. Pulmonary surfactant. The New England journal of medicine, 1971;284(21), pp. 1185-1193. doi: 10.1056/NEJM197105272842105.

6. Okada, T., Lee, B. W., Ogami, A., Oyabu, T. and Myojo, T. Inhalation of titanium dioxide (P25) nanoparticles to rats and changes in surfactant protein (SP-D) levels in bronchoalveolar lavage fluid and serum. Nanotoxicology, 2019;13, pp. 13961408 .

7. Takahashi, H., Kuroki, Y., Tanaka, H., Saito, T., Kurokawa, K., Chiba, H., Sagawa, A., Nagae, H. and Abe, S. Serum levels of surfactant proteins $\mathrm{A}$ and $\mathrm{D}$ are useful biomarkers for interstitial lung disease in patients with progressive systemic sclerosis. American journal of respiratory and critical care medicine, 2020;162(1), pp. 258-263. doi: 10.1164/ajrccm.162.1.9903014.

8. Saito, A., Kuronuma, K., Moniwa, K., Kodama, K., Takahashi, S., Takahashi, H. and Chiba, H. Serum surfactant protein A and D may be novel biomarkers of COVID-19 pneumonia severity. Research Square, 2020. doi: 10.21203/rs.3.rs-29567/v1.

9. Tong, M., Xiong, Y., Zhu, C., Xu, H., Zheng, Q., Jiang, Y., Zou, L., Xiao, X., Chen, F., Yan, X., Hu, C. and Zhu, Y. Serum surfactant protein D in COVID-19 is elevated and correlated with disease severity. BMC Infectious Diseases, 2021;21(1), p. 737. doi: 10.1186/s12879-021-06447-3.

10. Hahn, S. and Desai, S. C. Lower Lid Malposition: Causes and Correction. Facial Plastic Surgery Clinics of North America, 2016;24(2), pp. 163-171. doi: 10.1016/j.fsc.2015.12.006.

11. West, J. B. History of respiratory mechanics prior to World War II. Comprehensive Physiology, 2012;2(1), pp. 609-619. doi: 10.1002/cphy.c080112.

12. Barrow, R. E. and Hills, B. A. Surface tension induced by dipalmitoyl lecithin in vitro under physiological conditions. The Journal of physiology, 1979;297(0), pp. 217-227. doi: 10.1113/jphysiol.1979.sp013036.

13. Aung, H. H., Sivakumar, A., Gholani, S. K., Venkateswaran, S. P., Gorain, B. and Shadab, S. An Overview of the Anatomy and Physiology of the Lung. Nanotechnology-Based Targeted Drug Delivery Systems for Lung Cancer, 2019, pp. 1-20. doi:10.1016/B978-0-12-815720-6.00001-0 pp. 1-20. doi: 10.1016/B978-0-12-815720-6.00001-0.

14. Patwa, A. and Shah, A. Anatomy and physiology of respiratory system relevant to anaesthesia. Indian journal of anaesthesia, 
2015;59(9), pp. 533-541. doi: 10.4103/0019-5049.165849.

15. Lalley, P. M. The aging respiratory system--pulmonary structure, function and neural control. Respiratory physiology \& neurobiology, 2013;187(3), pp. 199-210. doi: 10.1016/j.resp.2013.03.012.

16. Fry, D. L. and Hyatt, R. E. Pulmonary mechanics. A unified analysis of the relationship between pressure, volume and gasflow in the lungs of normal and diseased human subjects. The American journal of medicine, 1960;29, pp. 672-689. doi: 10.1016/0002-9343(60)90100-5.

17. Arnal, J. M., Garnero, A., Saoli, M. and Chatburn, R. L. Parameters for Simulation of Adult Subjects During Mechanical Ventilation. Respiratory care, 2018;63(2), pp. 158-168. doi: 10.4187/respcare.05775.

18. Bakhtiar, A. and Maranatha, R.A. Acute Respiratory Syndrome. J. Respirasi, 2018;4, pp. 51-60.

19. Kosyreva, A., Dzhalilova, D., Lokhonina, A., Vishnyakova, P. and Fatkhudinoy, T. The Role of Macrophages in the Pathogenesis of SARS-CoV-2-Associated Acute Respiratory Distress Syndrome. Frontiers in Immunology, $2021 ; 12$, p. 1667. doi: 10.3389/fimmu.2021.682871.

20. Ragab, D. Eldin, H. S., Taeimah, M., Khattab, R. and Salem, R. The COVID-19 Cytokine Storm; What We Know So Far. Frontiers in Immunology, 2020;11, p. 1446. doi: 10.3389/fimmu.2020.01446.

21. Gattinoni, L., Chiumello, D., Caironi, P., Busana, M., Romitti, F., Brazzi, L. and Camporota, L. COVID-19 pneumonia: different respiratory treatments for different phenotypes? Intensive Care Medicine, 2020;46(6), pp. 1099-1102. doi: 10.1007/s00134-020-06033-2.

22. Han, S. and Mallampalli, R. K. The Role of Surfactant in Lung Disease and Host Defense against Pulmonary Infections. American Thoracic Society, 2015;12(5), pp. 765-774. doi: 10.1513/AnnalsATS.201411-507FR.

23. Schröder, H., Sollfrank, L., Paulsen, F., Bräuer, L. and Schicht, M. Recombinant expression of surfactant protein H (SFTA3) in Escherichia coli. Annals of Anatomy, 2016;208, pp. 129-134. doi: 10.1016/j.aanat.2016.05.006.

24. Bernhard, W. Lung surfactant: Function and composition in the context of development and respiratory physiology. Annals of Anatomy, 2016;208, pp. 146-150. doi: 10.1016/j.aanat.2016.08.003.

25. Wright, J. R. Immunomodulatory functions of surfactant. Physiological reviews, 1997;77(4), pp. 931-962. doi: 10.1152/physrev.1997.77.4.931.

26. Crouch, E., Hartshorn, K. and Ofek, I. Collectins and pulmonary innate immunity. Immunological reviews, 2000;173, pp. 5265. doi: 10.1034/j.1600-065x.2000.917311.x.

27. LeVine, A. M., Bruno, M. D., Huelsman, K. M., Ross, G. F., Whitsett, J. A. and Korfhagen, T. R. Surfactant protein Adeficient mice are susceptible to group B streptococcal infection. Journal of immunology, 1997;158(9), pp. 4336-4340.

28. LeVine, A. M., Kurak, K. E., Bruno, M. D., Stark, J. M., Whitsett, J. A. and Korfhagen, T. R. Surfactant protein-A-deficient mice are susceptible to Pseudomonas aeruginosa infection. American journal of respiratory cell and molecular biology, 1998;19(4), pp. 700-708. doi: 10.1165/ajrcmb.19.4.3254.

29. LeVine, A. M., Kurak, K. E., Wright, J. R., Watford, W. T., Bruno, M. D., Ross, G. F., Whitsett, J. A. and Korfhagen, T. R. Surfactant protein-A binds group B streptococcus enhancing phagocytosis and clearance from lungs of surfactant protein-Adeficient mice. American journal of respiratory cell and molecular biology, 1999;20(2), pp. 279-286. doi: 10.1165/ajrcmb.20.2.3303.

30. LeVine, A. M., Gwozdz, J., Stark, J., Bruno, M., Whitsett, J. and Korfhagen, T. Surfactant protein-A enhances respiratory syncytial virus clearance in vivo. The Journal of clinical investigation, 1999;103(7), pp. 1015-1021. doi: 10.1172/JCI5849.

31. Wu, H., Kuzmenko, A., Wan, S., Schaffer, L., Weiss, A., Fisher, J. H., Kim, K. S. and McCormack, F. X. Surfactant proteins A and D inhibit the growth of Gram-negative bacteria by increasing membrane permeability. The Journal of clinical investigation, 2003;111(10), pp. 1589-1602. doi: 10.1172/JCI16889.

32. McCormack, F. X., Gibbons, R., Ward, S. R., Kuzmenko, A., Wu, H. and Deepe Jr, G. S. Macrophage-independent fungicidal action of the pulmonary collectins. The Journal of biological chemistry, 2003;278(38), pp. 36250-36256. doi: 10.1074/jbc.M303086200.

33. Brinker, K. G., Garner, H. and Wright, J. R. Surfactant protein A modulates the differentiation of murine bone marrowderived dendritic cells. American journal of physiology, 2003;284(1), pp. L232-41. doi: 10.1152/ajplung.00187.2002.

34. Cheng, G., Ueda, T., Nakajima, H., Nakajima, A., Kinjyo, S., Motojima, S. and Fukuda, T. Suppressive effects of SP-A on ionomycin-induced IL-8 production and release by eosinophils. International archives of allergy and immunology, 1998;117 Suppl, pp. 59-62. doi: 10.1159/000053574.

35. Eisner, M. D., Parsons, P., Matthay, M. A., Ware, L. and Greene, K. Plasma surfactant protein levels and clinical outcomes in patients with acute lung injury. Thorax, 2003;58(11), pp. 983-988. doi: 10.1136/thorax.58.11.983.

36. Sorensen, G. L. Surfactant Protein D in Respiratory and Non-Respiratory Diseases. Frontiers in medicine, 2018;5, p. 18. doi: 10.3389/fmed.2018.00018.

37. Bridges, J. P., Davis, H. W., Damodarasamy, M., Kuroki, Y., Howles, G., Hui, D. Y. and McCormack, F. X. Pulmonary Surfactant Proteins A and D Are Potent Endogenous Inhibitors of Lipid Peroxidation and Oxidative Cellular Injury. Journal of Biological Chemistry, 2000;275(49), pp. 38848-38855. doi: 10.1074/jbc.M005322200.

38. Wert, S. E., Yoshida, M., LeVine, A. M., Ikegami, M., Jones, T., Ross, G. F., Fisher, J. H., Korfhagen, T. R. and Whitsett, J A. Increased metalloproteinase activity, oxidant production, and emphysema in surfactant protein D gene-inactivated mice. Proceedings of the National Academy of Sciences of the United States of America, 2000;97(11), pp. 5972-5977. doi: 10.1073/pnas.100448997.

39. Kerget, B., Kerget, F., Koçak, A. O., Araz, Ö., Uçar, E. Y. and Akgün, M. Are Serum Interleukin 6 and Surfactant Protein D 
Levels Associated with the Clinical Course of COVID-19? Lung, 2020;198(5), pp. 777-784. doi: 10.1007/s00408-020-003938.

40. Wiener, R. S., Cao, Y. X., Hinds, A., Ramirez, M. I. and Williams, M. C. Angiotensin converting enzyme 2 is primarily epithelial and is developmentally regulated in the mouse lung. Journal of cellular biochemistry, 2007;101(5), pp. 1278-1291. doi: $10.1002 / j \mathrm{jcb} .21248$.

41. Zhou, P., Yang, X., Wang, X., Hu, B., Zhang, K., Zhang, W., Si, H., Zhu, Y., Li, B., Huang, C., Chen, H., Chen, J., Luo, Y., Guo, H., Jiang, R., Liu, M., Chen, Y., Shen, X., Wang, X., Zheng, X., Zhao, K., Chen, Q., Deng, F., Liu, L., Yang, B., Zhan, F., Wang, Y., Xiao, G. and Shi, Z. A pneumonia outbreak associated with a new coronavirus of probable bat origin. Nature, 2020;579(7798), pp. 270-273. doi: 10.1038/s41586-020-2012-7.

42. Kurt, A., Turut, H., Acipayam, A., Kirbas, A., Yuce, S., Cure, M. C and Cure, E. Investigation of surfactant protein-D and interleukin-6 levels in patients with blunt chest trauma with multiple rib fractures and pulmonary contusions: a cross-sectional study in Black Sea Region of Turkey, BMJ open, 2016;6(10), pp. e011797-e011797. doi: 10.1136/bmjopen-2016-011797.

43. Arroyo, R. and Kingma, P. S. Surfactant protein D and bronchopulmonary dysplasia: a new way to approach an old problem, Respiratory Research, 2021;22(1), p. 141. doi: 10.1186/s12931-021-01738-4.

44. Zhang, T., Wu, Q. and Zhang, Z. Probable Pangolin Origin of SARS-CoV-2 Associated with the COVID-19 Outbreak. Current biology : CB, 2020;30(7), pp. 1346-1351.e2. doi: 10.1016/j.cub.2020.03.022 